\title{
SORÇÃO DA ATRAZINA EM SOLOS REPRESENTATIVOS DA SUB-BACIA DO RIO DAS MORTES - MG *
}

\author{
SAYONARAANDRADE DO COUTO MORENO ARANTES** \\ JOSÉ MARIA DE LIMA*** \\ JÚLIO CÉSAR AZEVEDO NÓBREGA**** \\ LUIZ ROBERTO GUIMARÃES GUILHERME*** \\ LUÍS GUSTAVO FERNANDES JULIÃO***** \\ ÉLEN ALVARENGA DE JESUS $S^{* \star * * *}$
}

\begin{abstract}
O objetivo deste trabalho foi avaliar a sorção do herbicida atrazina pelo método "batch" em amostras de Latossolo Vermelho Amarelo (LVA), Latossolo Vermelho (LV), Latossolo Vermelho Amarelo húmico (LVAh), Cambissolo Háplico (CX), Neossolo Flúvico (RU) e Latossolo Amarelo (LA). Esses solos são representativos da sub-bacia do Rio das Mortes, Minas Gerais (BRASIL) MG, que apresenta as maiores densidades populacionais e industriais da bacia do Alto Rio Grande (com grande importância econômica e social no estado). Isotermas de sorção foram ajustadas ao modelo linear e ao modelo matemático de Freundlich, sendo o ajuste linear dos dados melhor do que o ajuste ao modelo de Freundlich. Dentre os solos estudados, o RU foi o que apresentou o menor valor do coeficiente de partição $\left(K_{d}=1,34 \mathrm{~L} \mathrm{~kg}^{-1}\right)$, podendo ser o ambiente mais susceptível à contaminação por atrazina, seguido pelo $C X\left(K_{d}=2,16 \mathrm{~L} \mathrm{~kg}^{-1}\right)$. Os Latossolos foram os que apresentaram os maiores valores de $\mathrm{K}_{\mathrm{d}}$, variando de 2,86 $\mathrm{L} \mathrm{kg}^{-1}$ para o LA a $5,96 \mathrm{~L} \mathrm{~kg}^{-1}$ para o LVA, indicando menor potencial de movimento do produto para camadas mais profundas do perfil.
\end{abstract}

PALAVRAS-CHAVE: ATRAZINA; ISOTERMAS DE SORÇÃO; CONTAMINAÇÃO AMBIENTAL.

* $\quad$ Parte da Dissertação do primeiro autor, apresentada à Universidade Federal de Lavras (UFLA) para obtenção do grau de Mestre em Solos e Nutrição de Plantas. Financiado pela FAPEMIG e CNPq.

** Mestre em Solos e Nutrição de Plantas, Doutoranda em Solos e Nutrição de Plantas, Escola Superior de Agricultura "Luiz de Queiroz" (ESALQ), Piracicaba/SP (e-mail: samoreno@esalq.usp.br).

*** Professores, Doutores da UFLA, Lavras/MG (e-mail: jmlima@ufla.br; guilherm@ufla.br).

**** Professor, Doutor da Universidade Federal do Piauí (UFPI), Teresina, PI (e-mail: jnobrega@ufpi.br).

*****Alunos de Iniciação Científica, UFLA, Lavras/MG (e-mail: Igfj2002@yahoo.com.br; elenalvarenga @yahoo.com.br). 


\section{INTRODUÇÃO}

Os sistemas de produção agrícola empregam grandes quantidades de pesticidas, visando aumentar a quantidade e a qualidade dos produtos agrícolas a um custo razoável. O Brasil tornou-se um dos maiores consumidores de pesticidas do mundo, no entanto faltam pesquisas e informações mais detalhadas sobre a interação da maior parte desses produtos com os diferentes tipos de solos de regiões tropicais. Tais informações permitem otimizar o uso e evitar ou minimizar impactos desses produtos sobre o ambiente.

O herbicida atrazina, um dos mais consumidos no Brasil e no mundo, é utilizado em culturas de grande expressão econômica como milho, cana-de-açúcar e sorgo. Esse produto tem sido encontrado em águas de lençóis freáticos de vários países e por esse motivo seu uso foi proibido em 1990 na Alemanha e na Suécia (GRAYMORE, STAGNITTI e ALLISON, 2001).

A capacidade de sorção e dessorção dos pesticidas constitui o principal fator que influencia seu comportamento no solo. A sorção dos compostos aos constituintes do solo determina o potencial de transporte, transformação e efeitos biológicos dos pesticidas no ambiente (BARRIUSO et al., 1994). Segundo GUILHERME et al. (2000) os solos funcionam como "filtros" químicos e biológicos, reduzindo o impacto ambiental provocado por produtos químicos e evitando que resíduos dos pesticidas atinjam as águas superficiais e subsuperficiais. A sorção de moléculas de pesticidas pode variar enormemente em função das diferentes propriedades físicas e químicas do solo e do composto (MORAES e REZENDE, 1998).

Estudos têm mostrado que a matéria orgânica desempenha papel fundamental na sorção de pesticidas em solos. Segundo PICCOLO, CELANE e SIMONE (1992), a qualidade da matéria orgânica em razão dos seus grupos funcionais determina a sorção da atrazina em solos, uma vez que diferentes substâncias húmicas apresentam diferentes intensidades de sorção. Dentre os constituintes da matéria orgânica, o ácido húmico é responsável por cerca de 70\% da capacidade de sorção da atrazina (BARRIUSO et al., 1992). Outro fator físico-químico importante na sorção de pesticidas em solos é o pH. TRAGETTA et al. (1996) observaram sorção máxima da atrazina em ácido húmico e fúlvico próximo ao $\mathrm{pH} 3$, enquanto que para condições de pH normalmente encontradas nos solos (5 a 7) a sorção é menor.

As diferentes classes de solos apresentam comportamentos diferenciados no que diz respeito à contaminação ambiental por pesticidas. Segundo RESENDE et al. (1995), como os Latossolos estão presentes em maior extensão territorial são os solos mais empregados na agricultura, com formas de cultivo que demandam o emprego de maior volume de pesticidas. Entretanto, esses solos são relativamente menos erodíveis, armazenam maior quantidade de água e apresentam, via de regra, lençol freático mais profundo. Tal fato pode, de certa forma, minimizar o problema de contaminação ambiental causado pelo uso de pesticidas. Os solos de várzea, com lençol freático mais elevado e localização na parte baixa (receptora) da paisagem, constituem ambiente de maior risco (CURI, REZENDE e SANTANA, 1988), sendo mais susceptíveis à contaminação por poluentes.

Maior sorção da atrazina pelo solo reduz o potencial de contaminação resultante do seu uso intensivo e contínuo, característicos dos sistemas agrícolas modernos. É amplamente aceito que a variação na toxicidade inicial de herbicidas em solos depende da diferença na capacidade dos mesmos em sorver esses herbicidas (DAY, JORDAN e JOLLIFFE, 1968). Assim, conhecer a capacidade de sorção de pesticidas pelos solos em determinada região é fundamental para a seleção de práticas de manejo que possam reduzir os riscos de contaminação a outros compartimentos do ambiente.

A sub-bacia do Rio das Mortes é importante contribuinte da bacia do Alto Rio Grande. A principal atividade agrícola da região é a pecuária leiteira, embora tenha ocorrido significativo crescimento da área plantada com milho (Zea mays L.) (MARQUES, CURI e LIMA, 2002).

Com o objetivo de fornecer dados sobre a descarga de sedimentos dos três rios da bacia do Alto Rio Grande (Rio Capivari, Rio Grande e Rio das Mortes), SANTOS (1998) verificou maior contribuição 
do Rio das Mortes para a descarga de sedimentos e de sólidos suspensos sedimentáveis. Essa informação evidencia a importância do estudo do comportamento de poluentes nos solos da sub-bacia do Rio das Mortes.

Considerando a importância social e econômica da sub-bacia do Rio das Mortes, o objetivo deste estudo foi determinar a sorção da atrazina em classes de solos representativos dessa sub-bacia com o intuito de gerar informação para áreas com maior risco de impacto ambiental causado por esse herbicida.

\section{MATERIAL E MÉTODOS}

\subsection{CARACTERIZAÇÃO DA ÁREA DE ESTUDO}

A bacia do Alto Rio Grande, considerada a segunda maior bacia hidrográfica de Minas Gerais, abrange cerca de 87 mil km² dentro do estado. A bacia do Alto Rio Grande é constituída por três subbacias, a do Rio das Mortes (6 mil km²), a do Rio Capivari (2 mil km²) e a do Rio Grande (7 mil km²) (Figura 1). A sub-bacia do Rio das Mortes foi abordada no presente estudo por apresentar as maiores densidades populacionais e industriais do Alto Rio Grande. A mesma está localizada na região do Campo das Vertentes, a qual faz limite com as regiões Sul de Minas, Zona da Mata, Metropolitana e Oeste de Minas (GEOMINAS, 2006).

\section{FIGURA 1 - LOCALIZAÇÃO DA ÁREA DE ESTUDO NO ESTADO DE MINAS GERAIS E DISTRIBUIÇÃO DA REDE HIDROGRÁFICA}

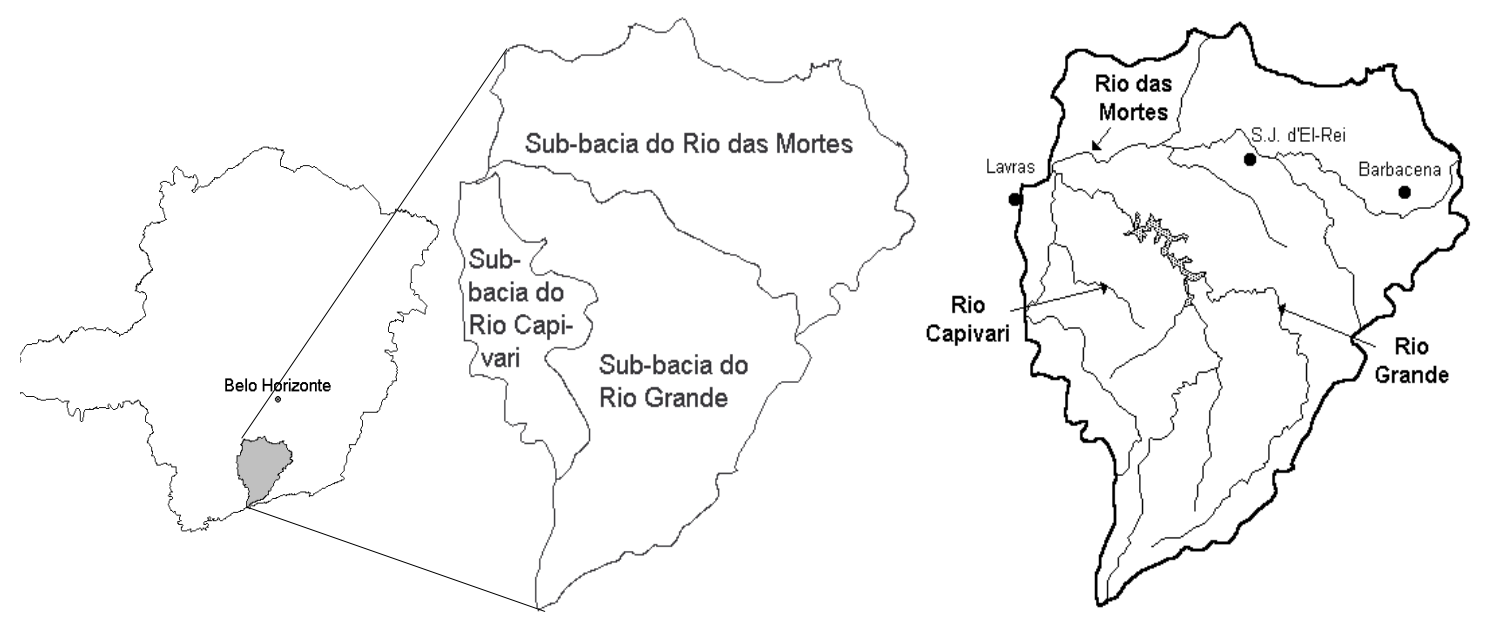

Fonte: MARQUES, CURI e LIMA (2002).

\subsection{SOLOS}

Foram utilizadas amostras da camada superficial $(0-20 \mathrm{~cm})$ de Latossolo Vermelho Amarelo (LVA), Latossolo Vermelho (LV), Cambissolo Háplico (CX), Neossolo Flúvico (RU), Latossolo Vermelho Amarelo húmico (LVAh) e Latossolo Amarelo (LA), classificados segundo o Sistema Brasileiro de Classificação de Solos (EMBRAPA, 1999). Esses solos são os mais representativos da sub-bacia do Rio das Mortes. Todos os pontos de amostragem estavam sob área de pastagem. 


\subsection{PREPARO E CARACTERIZAÇÃO DAS AMOSTRAS DE SOLOS}

Após a coleta, as amostras foram destorroadas, passadas em peneira de $2 \mathrm{~mm}$ de malha e deixadas secar ao ar para se obter a terra fina seca ao ar (TFSA). Efetuou-se a caracterização química e física dessas amostras em triplicata. Para a caracterização física dos solos foram determinados os teores de argila, silte e areia pelo método da pipeta (DAY, 1965). A areia total foi fracionada em conjunto de peneiras de malha $1 ; 0,5 ; 0,25$ e $0,105 \mathrm{~mm}$ em agitador mecânico, obtendo-se assim areia muito grossa, areia grossa, areia média, areia fina e areia muito fina de acordo com o sistema de classificação USDA (1975).

Para a caracterização química foram determinados os teores de cátions do complexo sortivo, pH em água, fósforo disponível e óxidos $\left(\mathrm{SiO}_{2}, \mathrm{Al}_{2} \mathrm{O}_{3}, \mathrm{Fe}_{2} \mathrm{O}_{3}, \mathrm{TiO}_{2}\right.$ e $\left.\mathrm{P}_{2} \mathrm{O}_{5}\right)$ extraídos pelo ataque sulfúrico, bem como os teores de carbono orgânico e matéria orgânica (EMBRAPA, 1997). Os resultados das análises físicas e químicas são apresentados nas Tabelas 1, 2 e 3.

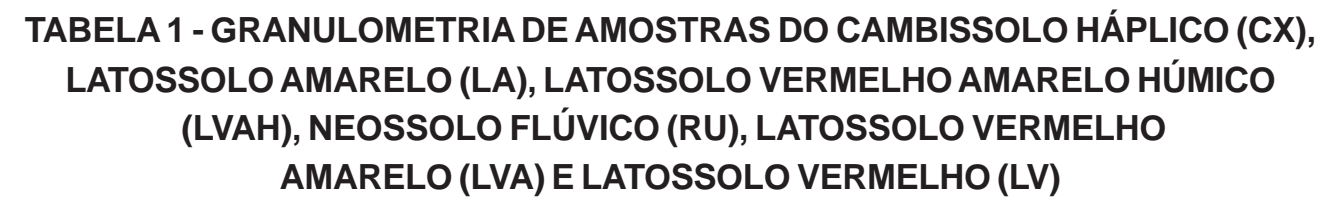

\begin{tabular}{ccccccccc}
\hline \multirow{2}{*}{ Solo } & Argila & Silte & Total & $\begin{array}{l}\text { Muito } \\
\text { Grossa }\end{array}$ & Grossa & Média & Fina & $\begin{array}{c}\text { Muito } \\
\text { Fina }\end{array}$ \\
\hline CX & 583 & 197 & 220 & 37 & 42 & 42 & 73 & 26 \\
LA & 327 & 360 & 313 & 37 & 67 & 56 & 87 & 66 \\
LVAh & 337 & 140 & 523 & 43 & 137 & 173 & 140 & 30 \\
RU & 233 & 337 & 430 & 10 & 26 & 50 & 197 & 147 \\
LVA & 530 & 147 & 323 & 37 & 93 & 83 & 87 & 23 \\
LV & 340 & 193 & 467 & 31 & 71 & 122 & 193 & 50 \\
\hline
\end{tabular}

TABELA 2 - TEORES DE ÓXIDOS EXTRAÍDOS PELO ATAQUE SULFÚRICO E ÍNDICES DE INTEMPERISMO (Ki E Kr) DE AMOSTRAS DO CAMBISSOLO HÁPLICO (CX), LATOSSOLO AMARELO (LA), LATOSSOLO VERMELHO AMARELO HÚMICO (LVAH), NEOSSOLO FLÚVICO (RU), LATOSSOLO VERMELHO AMARELO (LVA) E LATOSSOLO VERMELHO (LV)

\begin{tabular}{|c|c|c|c|c|c|c|c|}
\hline Solo & $\mathrm{SiO}_{2}$ & $\mathrm{Al}_{\mathbf{2}} \mathrm{O}_{3}$ & $\mathrm{Fe}_{2} \mathrm{O}_{3}$ & $\mathrm{TiO}_{2}$ & $\mathbf{P}_{2} \mathbf{O}_{5}$ & $\mathbf{K i}$ & $\mathbf{K r}$ \\
\hline \multicolumn{8}{|c|}{ 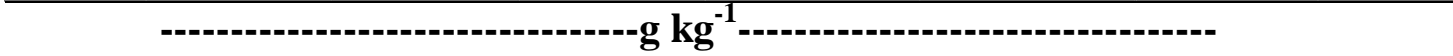 } \\
\hline CX & 239,7 & 257,1 & 135,8 & 27,7 & 0,7 & 1,6 & 1,2 \\
\hline LA & 80,8 & 284,9 & 125,1 & 26,0 & 0,8 & 0,5 & 0,4 \\
\hline LVAh & 120,0 & 181,6 & 41,7 & 8,4 & 0,6 & 1,1 & 0,9 \\
\hline RU & 184,7 & 155,2 & 64,9 & 16,9 & 1,1 & 2,0 & 1,6 \\
\hline LVA & 170,2 & 221,9 & 112,6 & 19,6 & 1,1 & 1,3 & 0,9 \\
\hline LV & 111,6 & 207,8 & 85,6 & 18,4 & 0,5 & 0,9 & 0,7 \\
\hline
\end{tabular}




\section{TABELA 3 - CARACTERIZAÇÃO QUÍMICA DE AMOSTRAS DO CAMBISSOLO HÁPLICO (CX), LATOSSOLO AMARELO (LA), LATOSSOLO VERMELHO AMARELO HÚMICO (LVAH), NEOSSOLO FLÚVICO (RU), LATOSSOLO VERMELHO (LVA) E LATOSSOLO VERMELHO (LV)}

\begin{tabular}{|c|c|c|c|c|c|c|c|c|c|c|c|c|c|}
\hline Solo & $\mathbf{p H}$ & $\mathbf{P}$ & $\mathbf{K}^{+}$ & $\mathrm{Ca}^{2+}$ & $\mathrm{Mg}^{2+}$ & $\overline{\mathbf{A l}^{3+}}$ & $\mathbf{S}$ & $\mathbf{t}$ & $\mathbf{T}$ & V & $\mathbf{m}$ & MO & $\mathrm{CO}$ \\
\hline & \multicolumn{3}{|c|}{$--m g ~ d m^{-3}--$} & \multicolumn{6}{|c|}{ - } & \multicolumn{2}{|c|}{$---\%$} & \multicolumn{2}{|c|}{$---\mathrm{g} \mathrm{kg}^{-1}-\cdots$} \\
\hline $\mathrm{CX}$ & 5,1 & 1,4 & 39,0 & 0,7 & 0,2 & 0,9 & 1,0 & 1,9 & 8,9 & 11,2 & 47,0 & 45,5 & 26,4 \\
\hline LA & 6,0 & 1,4 & 97,0 & 2,7 & 1,7 & 0,0 & 4,7 & 4,7 & 7,6 & 61,6 & 0,0 & 46,8 & 27,2 \\
\hline LVAh & 5,4 & 10,4 & 48,0 & 2,2 & 0,6 & 0,8 & 2,9 & 3,7 & 11,7 & 24,9 & 22,0 & 38,6 & 22,4 \\
\hline RU & 5,8 & 10,8 & 179,0 & 3,2 & 2,1 & 0,2 & 5,8 & 6,0 & 9,0 & 64,3 & 3,0 & 34,4 & 20,0 \\
\hline LVA & 5,1 & 3,7 & 30,0 & 1,5 & 0,3 & 1,1 & 1,9 & 3,0 & 11,7 & 16,1 & 37,0 & 41,3 & 24,0 \\
\hline LV & 5,2 & 1,4 & 75,0 & 0,8 & 0,2 & 0,5 & 1,2 & 1,7 & 7,5 & 15,9 & 30,0 & 37,2 & 21,6 \\
\hline
\end{tabular}

pH em água: relação $(1: 2,5) ; \mathrm{S}=$ soma de bases trocáveis; $\mathrm{t}=\mathrm{CTC}$ efetiva; $\mathrm{T}=\mathrm{CTC}$ a pH 7,0; $\mathrm{V}=$ índice de saturação de bases; $m$ = índice de saturação de alumínio; $\mathrm{MO}$ = matéria orgânica; $\mathrm{CO}$ = carbono orgânico.

\subsection{ESTUDO DE SORÇÃO}

A sorção dos solos foi avaliada pelo método "batch". Para o estabelecimento de isotermas de sorção foram colocados $2,0 \mathrm{~g}$ de cada amostra de solo em frascos de centrífuga. Adicionaram-se $20,0 \mathrm{~mL}$ de solução da atrazina nas concentrações de $0 ; 0,125 ; 0,25 ; 0,5 ; 1,0 ; 2,5 ; 5,0 ; 10,0 ; 20,0$; 25,0 e $30,0 \mathrm{mg} \mathrm{L}^{-1}$, preparadas em $\mathrm{CaCl}_{2} 2 \mathrm{H}_{2} \mathrm{O}\left(0,01 \mathrm{~mol} \mathrm{~L}^{-1}\right)$ (AMARAL, 2004). As suspensões foram agitadas durante 24 horas e centrifugadas a $16700 \mathrm{~g}$ por 10 minutos. O sobrenadante foi filtrado em membrana $0,20 \mu \mathrm{m}$ e a concentração da atrazina quantificada por cromatografia a líquido de alta eficiência (CLAE).

$\mathrm{Na}$ análise cromatográfica empregou-se água ultrapura e metanol $(80: 20 / \mathrm{V}: \mathrm{V})$ como fase móvel, temperatura de operação da coluna de $50^{\circ} \mathrm{C}$, detector DAD $(222 \mathrm{~nm})$, após separação em coluna de fase reversa C18 $(5 \mu \mathrm{m} ; 3,0 \times 25 \mathrm{~cm})$. Usou-se fluxo de 0,8 $\mathrm{mL} \mathrm{min}^{-1}$ até 2,2 min, reduzido para $0,5 \mathrm{~mL} \mathrm{~min}^{-1}$ até 2,6 min e retornando a $0,8 \mathrm{~mL} \mathrm{~min}^{-1}$ até 3 minutos. Os resultados foram comparados com curva-padrão construída a partir do padrão analítico da atrazina, grau de pureza 99,5\%, cedido pela Novartis.

Quantificou-se a sorção da atrazina pelos solos conforme a expressão 1:

$$
x / m=(C i-C e) * v / m
$$

Em que:

$\mathrm{x} / \mathrm{m}=$ quantidade de atrazina sorvida $\left(\mathrm{mg} \mathrm{kg}^{-1}\right)$;

$\mathrm{C}_{\mathrm{i}}$ e $\mathrm{C}_{\mathrm{e}}=$ concentrações de atrazina inicial e no equilíbrio $\left(\mathrm{mg} \mathrm{L}^{-1}\right)$;

$\mathrm{v}=$ volume da solução de atrazina (L); e

$\mathrm{m}=$ massa de solo $(\mathrm{kg})$.

Os resultados da sorção da atrazina foram ajustados à equação linearizada de Freundlich (Equação 2):

Na qual:

$$
\log (x / m)=\log K_{f}+1 / n \log C e
$$

$\mathrm{x} / \mathrm{m}$ = quantidade de atrazina sorvida por massa de solo $\left(\mathrm{mg} \mathrm{kg}^{-1}\right)$;

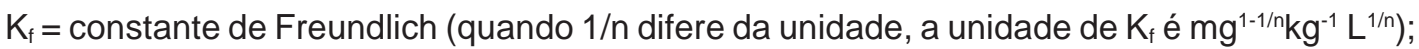

$\mathrm{Ce}=$ concentração da atrazina na solução em equilíbrio com o solo; e

$1 / n=$ grau de linearidade da isoterma de sorção. 
Foram calculados também o coeficiente de partição $\left(K_{d}\right)$ (Equação 3) e o coeficiente de partição em relação ao carbono orgânico do solo ( $\left.\mathrm{K}_{\mathrm{oc}}\right)$ (Equação 4):

$$
\begin{gathered}
k_{d}=(x / m) / C e \\
K_{o c}=\left(K_{d} / C\right) * 1000
\end{gathered}
$$

Em que:

$\mathrm{C}=$ teor de carbono orgânico do solo $\left(\mathrm{g} \mathrm{kg}^{-1}\right)$.

Para comparar os valores do coeficiente de Freundlich $\left(\mathrm{K}_{\mathrm{f}}\right)$ e o coeficiente de distribuição $\left(\mathrm{K}_{\mathrm{d}}\right)$ aplicou-se o teste de Tukey, a 5\% de significância, de acordo com PIMENTEL-GOMES e GARCIA (2002).

\section{RESULTADOS E DISCUSSÃO}

Os resultados de sorção da atrazina ajustados ao modelo de Freundlich e à equação linear são apresentados na Tabela 4.

O ajuste à equação linear produziu valores de $\mathrm{R}^{2}$ ligeiramente superiores que a equação de Freundlich. Tal resultado sugere que os sítios de sorção envolvidos são relativamente homogêneos para a faixa de concentração da atrazina avaliada. Esse comportamento indica que nas seis classes de solos estudadas, a sorção da atrazina aumenta linearmente com a elevação da sua concentração na solução.

\section{TABELA 4 - AJUSTE DOS DADOS DE SORÇÃO DE ATRAZINA AO MODELO DE FREUNDLICH E EQUAÇÃO LINEAR PARA SOLOS REPRESENTATIVOS DA

\begin{tabular}{|c|c|c|c|c|c|c|c|c|}
\hline \multirow{2}{*}{ Solo } & \multicolumn{3}{|c|}{ Equacão de Freundlich } & \multicolumn{3}{|c|}{ Equação linear } & \multirow{2}{*}{ pH } & \multirow{2}{*}{ MO } \\
\hline & $\mathbf{K}_{\mathbf{f}}$ & $1 / n$ & $\mathbf{R}^{2}$ & $\mathbf{K}_{\mathbf{d}}$ & $\mathbf{R}^{2}$ & $K_{\text {oc }}$ & & \\
\hline & $\mathrm{mg}^{1-1 / n} \mathrm{~kg}^{-1} \mathrm{~L}^{1 / \mathrm{n}}$ & & & $\mathrm{L} \mathrm{kg}^{-1}$ & & $\mathrm{~L} \mathrm{~kg}^{-1}$ & & $\mathrm{~g} \mathrm{~kg}^{-1}$ \\
\hline $\mathrm{CX}$ & $2,18 \pm 1,10$ & 0,93 & 0,81 & $2,16 \pm 0,31$ & 0,98 & $81,86 \pm 11,76$ & 5,1 & 45,5 \\
\hline LA & $3,25 \pm 0,33$ & 0,88 & 0,90 & $2,86 \pm 0,26$ & 0,98 & $105,15 \pm 9,43$ & 6,0 & 46,8 \\
\hline LVAh & $4,28 \pm 0,54$ & 0,87 & 0,94 & $3,03 \pm 0,12$ & 0,97 & $135,27 \pm 5,23$ & 5,4 & 38,6 \\
\hline $\mathrm{RU}$ & $2,81 \pm 0,39$ & 0,74 & 0,90 & $1,34 \pm 0,11$ & 0,90 & $67,00 \pm 5,21$ & 5,8 & 34,4 \\
\hline LVA & $4,89 \pm 0,59$ & 1,04 & 0,93 & $5,96 \pm 0,07$ & 0,96 & $248,33 \pm 2,71$ & 5,1 & 41,3 \\
\hline LV & $3,64 \pm 0,34$ & 1,05 & 0,88 & $4,87 \pm 0,15$ & 0,97 & $225,46 \pm 6,87$ & 5,2 & 37,2 \\
\hline
\end{tabular} SUB-BACIA DO RIO DAS MORTES}

$\mathrm{K}_{\mathrm{f}}=$ Constante de Freundlich para sorção; $1 / \mathrm{n}=$ Grau de linearidade da isoterma; $\mathrm{K}_{\mathrm{d}}=$ Coeficiente de partição; $\mathrm{K}_{\mathrm{oc}}=$ Coeficiente de partição normalizado para carbono orgânico; $\mathrm{MO}$ = Matéria orgânica. * pH em água.

Na literatura, o modelo matemático de Freundlich têm sido aplicado com eficiência (YAMANE e GREEN, 1972; SWANSON e DUTI, 1973; LAIRD et al., 1992; PICCOLO, CELANO e SIMONE 1992; BARRIUSO, 1994; CELIS et al., 1997; MORAES e REZENDE, 1998; GOMES, DICK e SOUZA, 2002).

Para GOMES, DICK e SOUZA (2002) o K é usualmente calculado nos estudos de sorção de herbicidas considerando as interações herbicida-solo não somente como fenômeno de superfície, mas como fenômeno que ocorre de maneira homogênea em todo o volume do solo $\left(K_{d}\right)$ ou da matéria orgânica ( $\mathrm{K}_{\mathrm{oc}}$ ). Segundo HERWING et al. (2001), a isoterma linear é adequada para substâncias pouco ou medianamente solúveis em água (como é o caso da atrazina). 
O valor do $\mathrm{K}_{\mathrm{d}}$ para os solos estudados variou de 1,34 a 5,96 $\mathrm{L} \mathrm{kg}^{-1}$ (Tabela 4), que se deve a diferenças nas características físicas (Tabela 1) e químicas (Tabela 2 e 3 ) dos solos.

O Neossolo Flúvico (RU) apresentou o mais baixo valor de $K_{d}$ em razão de menores teores de matéria orgânica (Tabela 3) e argila (Tabela 1) presentes nesse solo. Segundo KOSKINEN e HARPER (1990), a área superficial específica da argila relacionada à capacidade de troca catiônica confere ao solo a propriedade de bom adsorvente de pesticidas. $\mathrm{O}$ valor de $\mathrm{pH}$ desse solo, relativamente mais elevado, também contribuiu para a menor sorção de atrazina. Para CELIS et al. (1997) a molécula se torna mais neutra em pH mais elevado diminuindo a sua protonação e, conseqüentemente, a sua sorção ao solo.

Solos de várzea, por apresentarem lençol freático mais elevado e estarem presentes na parte mais baixa da paisagem, são considerados ambientes mais propensos à contaminação ambiental. Dentre os solos da sub-bacia do Rio das Mortes, o Neossolo Flúvico pode ser considerado o mais frágil com relação a possíveis impactos ambientais causados pelo herbicida atrazina se utilizado mais intensivamente. PESSOA et al. (2003) estudaram áreas de exposição ao risco de contaminação de águas subterrâneas pelos herbicidas atrazina, diuron e tebutiuron. Verificaram que o Neossolo Quartzarênico, com lençol freático mais superficial apresenta maior risco de exposição aos herbicidas estudados. Situação oposta foi encontrada para os Latossolos, principalmente o Latossolo Vermelho Amarelo (LVA) e o Latossolo Vermelho (LV), com maiores valores de $K_{d}\left(5,96\right.$ e 4,87 $\left.\mathrm{L} \mathrm{kg}^{-1}\right)$.

Os Latossolos (de ocorrência expressiva na sub-bacia do Rio das Mortes) apresentam lençol freático mais profundo e maior capacidade de sorção da atrazina, conforme verificado no presente estudo. Embora menos susceptíveis à contaminação pelo produto, o manejo correto e racional da atrazina e de outros pesticidas torna-se necessário para evitar possíveis impactos sobre outros compartimentos do ambiente e animais.

O Cambissolo Háplico (CX) apresentou valor intermediário de $\mathrm{K}_{d}$ entre o Neossolo Flúvico e os Latossolos estudados. Segundo RESENDE et al. (1995), os Cambissolos demonstram comportamento intermediário no que diz respeito à contaminação ambiental.

Apesar da matéria orgânica ser considerada a principal responsável pela capacidade sortiva da atrazina pelos solos, no presente estudo não se verificou correlação entre o teor de matéria orgânica e a capacidade sortiva dos mesmos. Tal fato contradiz os resultados obtidos por BARRIUSO et al., 1992; GOMES, DICK e SOUZA, 2002 e PRATA, 2002. Esse comportamento se deve, provavelmente, ao uso de diferentes classes de solos com atributos físicos e químicos distintos e interação entre as fases orgânica e mineral dos solos.

O Latossolo Amarelo (LA) apresentou o maior teor de matéria orgânica, porém seu $K_{d}$ foi praticamente igual ao do Latossolo Vermelho Amarelo húmico (LVAh) e mais baixo que o dos demais Latossolos estudados (LVA e LV). Esse resultado indica que além do teor, o tipo e a relação da matéria orgânica com os minerais dos solos assumem importância na sorção da atrazina. Deve-se destacar que no presente estudo, os solos amostrados encontravam-se sob diferentes espécies forrageiras o que pode acarretar diferenças na qualidade da matéria orgânica dos solos.

Apesar da matéria orgânica ser considerada o principal responsável pela sorção de pesticidas, poucos estudos são realizados levando em conta as características químicas e físicas das substâncias húmicas. PICCOLO, CELANO e SIMONE (1992) pesquisaram a sorção da atrazina em três diferentes substâncias húmicas e verificaram variação na sorção do produto em função dos diferentes tipos de substâncias húmicas estudadas. CELIS et al. (1998) relataram que a contribuição da matéria orgânica na sorção de atrazina e simazina pode ser reduzida pela associação de ácidos húmicos com óxidos de ferro. Quando há formação de complexos ácidos húmicos-argila ou ácidos húmicos-óxidos, os ácidos húmicos ficam impedidos de adquirir conformação mais aberta e, conseqüentemente, de expor grande parte de seus sítios hidrofóbicos, diminuindo a sorção de moléculas nesse componente orgânico.

Os valores de $K_{d}$ encontrados neste estudo $\left(1,34\right.$ a 5,96 $\left.\mathrm{L} \mathrm{kg}^{-1}\right)$ enquadraram-se na faixa de valores citados na literatura. Sob as mesmas condições experimentais, CORREIA (2000) encontrou para atrazina em solo Podzólico Vermelho Amarelo valores de $K_{d}$ variando de 1,71 a 2,58 L kg-1. LIMA 
(2004) porém, estudando a sorção da atrazina em Latossolo Vermelho Amarelo distrófico e em Latossolo Vermelho distrófico sob efeito de calagem e fosfatagem obteve valores mais elevados de $K_{d}(11,42 a$ $\left.16,65 \mathrm{~L} \mathrm{~kg}^{-1}\right)$.

O resultado do coeficiente de partição normalizado para carbono orgânico $\left(\mathrm{K}_{\circ c}\right)$ caracteriza a atrazina como herbicida de alta mobilidade no Neossolo Flúvico e no Cambissolo Háplico. Segundo BEWICK (1994), herbicidas com valores de $\mathrm{K}_{\mathrm{oc}}$ entre 50 e $100 \mathrm{~L} \mathrm{~kg}^{-1}$ apresentam alta mobilidade no perfil do solo.

\title{
4 CONCLUSÃO
}

O Neossolo Flúvico, seguido pelo Cambissolo Háplico foram os solos com menor capacidade de sorção de atrazina e, portanto, mais susceptíveis à contaminação por esse herbicida.

Os Latossolos, de modo geral, apresentaram os maiores valores de $K_{d}$, indicando que são "melhores filtros" na retenção da atrazina.

\begin{abstract}
ATRAZINE SORPTION IN REPRESENTATIVE SOILS OF THE RIO DAS MORTES SUB-BASIN AREA

The objective of the present work was to evaluate the sorption of atrazine, using the "batch" method, in samples of a Red Yellow Latosol (LVA), a Red Latosol (LV), a humic Red Yellow Latosol (LVAh), a Haplitic Cambisol (CX), a Fluvic Neosol (RU), and a Yellow Latosol (LA). These soils are representative of the Rio das Mortes - MG sub-basin area, which has the largest population and industrial density of the Alto Rio Grande basin area (with great socio-economic importance in Minas Gerais State). The sorption isotherms were adjusted to the linear model and to the mathematical Freundlich model, being the data better adjusted to the linear than to the Freundlich model. Among the studied soils, RU presented the smallest value for the partition coefficient $\left(\mathrm{K}_{\mathrm{d}}=1.34 \mathrm{~L} \mathrm{~kg}^{-1}\right)$, therefore, it can be the most susceptible soil for atrazine leaching, followed by $C X\left(K_{d}=2.16 \mathrm{~L} \mathrm{~kg}^{-1}\right)$. Latosols showed the largest values of $\mathrm{K}_{\mathrm{d}}$, ranging from $2.86 \mathrm{~L} \mathrm{~kg}^{-1}$, for $\mathrm{LA}$, to $5.96 \mathrm{~L} \mathrm{~kg}^{-1}$ for LVA, which indicate higher sorption in Latosols, showing evidences for a lower potential for atrazine mobility to deeper layers in these soil profiles.
\end{abstract}

KEY-WORDS: ATRAZINE; SORPTION ISOTHERMS; ENVIRONMENTAL CONTAMINATION.

\section{REFERÊNCIAS}

1 AMARAL, L.C.S. Sorção e mobilidade de atrazina em latossolos da região de Lavras - MG. Lavras, 2004. 158 p. Tese (Doutorado) - Universidade Federal de Lavras.

2 BARRIUSO, E.; FELLER, C.; CALVET, R.; CERRI, C. Sorption of atrazine, terbutryn and 2,4-D herbicides in two Brazilian Oxisols. Geoderma, v.53, n.1/2, p.155-167, 1992.

3 BARRIUSO, E. Atrazine desorption from smectites. Soil Science Society America Journal, v.58, n.6, p.1632-1638, 1994.

4 BEWICK, D. The mobility of pesticides in soil - studies to prevent groundwater contamination. In: BORNER, H. Pesticides in ground and surface water. Heidelberg: Springer-Verlag, 1994. p.61.

5 CELIS, R.; CORNEJO, J.; HERMOSIN, M.C.; KOSKINEN, W.C. Sorption of atrazine and simazine by model associations of soil colloids. Soil Science Society America Journal, v.62, n.1, p.165-171, 1998.

6 CELIS, R.; CORNEJO, J.; HERMOSIN, M.C.; KOSKINEN, W.C. Sorption-desorption of atrazine and simazine by model soil colloidal components. Soil Science Society of America Journal, v.61, n.2, p. 436-443, 1997.

7 CORREIA, F.V. Distribuição e degradação do herbicida atrazina em solo Podzólico Vermelho Amarelo sob condições de clima tropical úmido. Lavras, 2000. 83 p. Dissertação (Mestrado)-Universidade Federal de Lavras, 
8 CURI, N.; RESENDE, M.; SANTANA, D.P. Solos de várzea de Minas Gerais. Informe Agropecuário, Belo Horizonte, v.13, n.152, p.3-10, 1988.

9 DAY, B.E.; JORDAN, L.S.; JOLLIFFE. The influence of soil characteristics on the adsorption and phytotoxicity of simazine. Weed Science, v.16, n.2, p.209-213, 1968.

10 DAY, P.R. Particle fractionation and particle-size analysis. In: BLACK, C.A. (Ed.). Methods of soil analysis. Madison: American Society of Agronomy, 1965. v.1, p.545-566.

11 EMBRAPA. Empresa Brasileira de Pesquisa Agropecuária. Centro Nacional de Pesquisa de Solo. Manual de métodos de análises de solo. 2.ed. Rio de Janeiro, 1997. 212 p.

12 EMBRAPA. Empresa Brasileira de Pesquisa Agropecuária. Centro Nacional de Pesquisa de Solo. Sistema brasileiro de classificação de solos. Rio de Janeiro, 1999. 412 p.

13 GEOMINAS. Geoprocessamento em Minas Gerais. 2006. Disponível em: <http:// www.geominas.mg.gov.br>. Acesso em: 27 jan. 2006.

14 GOMES, J.; DICK, D.P.; SOUZA, R.F. Sorção de atrazina em Cambissolo Húmico do Rio Grande do Sul sob vegetação nativa. Revista Brasileira de Ciência do Solo, v.26, n.2, p.521-528, 2002.

15 GRAYMORE, M.; STAGNITTI, F.; ALLISON, G. Impacts of atrazine in aquatic ecosystems. Environment International, v.26, n.7-8, p.483-495, 2001.

16 GUILHERME, L.R.G.; SILVA, M.L.N.; LIMA, J.M.; RIGITANO, R.L. de O. Contaminação de microbacia hidrográfica pelo uso de pesticidas. Informe Agropecuário, Belo Horizonte v.21, n.207, p.40-54, 2000.

17 HERWING, U. et al. Physicochemical interactions between atrazine and clay minerals. Applied Clay Science, v.18, p.211-222, 2001.

18 KOSKINEN, W.C.; HARPER, S. The retention process: mechanisms. In: CHENG, H.H. et al. (Ed.). Pesticides in the soil environment: processes, impacts, and modeling. Madison: Soil Science Society of America, 1990. p.51-78.

19 LAIRD, D.A.; BARRIUSO, E.; DOWDY, R.H.; KOSKINEN, W.C. Adsorption of atrazine on smectites. Soil Science Society America Journal, v.56, n.1, p.62-67. 1992.

20 LIMA, D.M. Sorção e deslocamento miscível da atrazina em amostras de latossolos. Lavras, 2004. 66 p. Dissertação (Mestrado) - Universidade Federal de Lavras.

21 MARQUES, J.J.G.S.M.; CURI, N.; LIMA, J.M. Recursos ambientais do Alto Rio Grande, Minas Gerais: problemas, fisiografia e guia de excursão técnica. Lavras: UFLA/FAEPE, 2002. 33 p.

22 MORAES, S.L.; REZENDE, M.O. de O. Comportamento sortivo dos herbicidas s-triazinas em solo e em ácidos húmicos. Pesticidas: revista de ecotoxicologia e meio ambiente, v.8, p.157-170, 1998.

23 PESSOA, M.C.P.Y.; GOMES, M.A.F.; NEVES, M.C.; SOUZA, M.D. de. Identificação de áreas de exposição ao risco de contaminação de águas subterrâneas pelos herbicidas atrazina, diuron, e tebutiuron. Pesticidas: revista de ecotoxicologia e meio ambiente, v.13, p. 111-122, 2003.

24 PICCOLO, A.; CELANO, G. SIMONE, C. de. Interactions of atrazine with humic substances of different origins and their hydrolysed products. The Science of the Total Environment, v.117-118, p.403-412, 1992.

25 PIMENTEL-GOMES, F.; GARCIA, C.H. Estatística aplicada a experimentos agronômicos e florestais. Piracicaba: FEALQ, 2002. 309 p.

26 PRATA, F. Comportamento do glifosato no solo e deslocamento miscível de atrazina. Piracicaba, 2002. 149 p. Tese (Doutorado) - Escola Superior de Agricultura "Luiz de Queiroz".

27 RESENDE, M.; CURI, N.; REZENDE, S.B. de., CORRÊA, G.F. Pedologia: base para distinção de ambientes. Viçosa: NEPUT, 1995. 304 p. 
28 SANTOS, E.H.M. Descarga de sedimentos transportados em suspensão por três rios da Bacia Hidrográfica do Alto Rio Grande. Lavras, 1998. 58 p. Dissertação (Mestrado em Irrigação e Drenagem) - Universidade Federal de Lavras.

29 SWANSON, R.A.; DUTT, G.R. Chemical and physical processes that affect atrazine and distribution in soil system. Soil Science Society America Proceedings, v.37, n.5, p.872-876, 1973.

30 TRAGETTA, D.G.; VAZ, C.M.P.; MACHADO, S.A.S.; VIEIRA, E.M.; MARTIN-NETO, L. Mecanismos de sorção da atrazina em solos: estudos espectroscópicos e polarográficos. Comunicado Técnico, São Carlos, n.14, p. 1-7, dez. 1996.

31 USDA. United States Department of Agriculture. Soil taxonomy: a basic system of soil classification for making and interpreting soil surveys. Washington, 1975. 436 p.

32 YAMANE, V.K.; GREEN, R.E. Adsorption of ametryne and atrazine on an Oxisol, montmorillonite, and charcoal in relation to $\mathrm{pH}$ and solubility effects. Soil Science and Society America Proceedings, v.36, n.1, p.58-64, 1972.

\section{AGRADECIMENTOS}

À Fundação de Amparo à Pesquisa do Estado de Minas Gerais - FAPEMING, pelo suporte financeiro para a realização da pesquisa e ao Conselho Nacional de Desenvolvimento Científico e Tecnológico CNPQ, pela concessão da bolsa durante a realização do mestrado. 